\title{
jur-pc: Ausbildungsrelevant?
}

Im Computerraum der Juristen in Saarbrücken liegen immer jur-pc-Exemplare aus. Selten sieht man Studenten zögern, ob sie zugreifen sollen. Einmal war es aber doch so. Und da ich gerade dabei stand, fragte mich der zögernde Kommilitone: "Ist das ausbildungsrelevant?" Ich sagte " ja”, nahm mir aber vor, diese Überzeugung noch einmal zu überprüfen. Die folgende Übersicht ist das Ergebnis dieser Selbstprüfung.

Neben dem Lesen gehört das Schreiben zum Studienalltag. Folglich hat jur-pc die Entwicklung der Textverarbeitungsprogramme begleitet, wobei besonders die Verbindung mit den Besonderheiten der juristischen Texte einen Prüf- und Darstellungsmaßstab abgab (vgl. als aktuelles Beispiel Knop zur juristischen Seminararbeit und Winword, jur-pc 1995, S. 3417-3432 u. 3474-3484). Im übrigen sollen die Texte möglichst fehlerfrei sein. Die EDV. unterstützt dieses Bemühen, teils schon im Rahmen der Textverarbeitung, teils durch eigenständige Zusatzprogramme. Auch dazu soll man sich in jur-pc orientieren können (vgl. z. B. Drosdeck, PRIMUS - eine Rechtschreibkontrolle für Juristen, jur-pc 1990, S. 792-798). Die juris-Nutzung wird immer mehr Alltagsbestandteil des Jura-Studiums. Wie man hört, haben alle Fakultäten mit CIP-Räumen inzwischen Nutzungsverträge. Das nötige juris-Benutzungs-Know-How konnte man stets aus jur-pc beziehen (das begann mit Böttcher, juris - nur etwas für Informatik-Freaks?, jur-pc 1989, S. 266-274; S. 335-345; vgl. auch noch Jabn, juris - (nicht nur) für Einsteiger, jur-pc 1994, S. 2685-2694, 2717-2725).

CD-ROM's sind jetzt wie Bücher Handwerkszeug im Studium. Folglich sind sie im Prinzip wie Bücher zu rezensieren (vgl. z. B. zum elektronischen Schönfelder die zeitnahe Rezension in jur-pc 1994, S. 2740-2746 und das aktuelle Interview zum gleichen Thema, jur-pc 1994, S. 2748-2751); oder zur in der Ausbildung oft nachgefragten Leitsatzkartei jur-pc 1994, S. 2530-2533). Einmal jährlich erscheint der jur-pc CD-ROM-Digest mit einer Übersicht über alle deutschen juristischen CD-ROM's, da werden die ausbildungsrelevanten dann notwendigerweise mit behandelt. Wo elektronische Lernsysteme entstanden, war jur-pc aktuell dabei (vgl. z. B. Günther, Alpmann ESchmidt elektronisch, jur-pc 1990, 759-766; oder zu Krobn's Staatshaftungsskript mit Diskettenbeilage Konzelmann, jur-pc 1994, S. 2902-2903); oder Knoop, Das HGBLernprogramm Handelsrecht, jur-pc 1994, S. 2942-2945). Hin und wieder haben Autoren auch eigene Lernprogramme in jur-pc vorgestellt und auf Diskette beigegeben, so etwa Steins (vgl. jur-pc 1994, S. 2726-2729) mit eTutor, einem (immer noch) empfehlenswerten Lernprogramm zum Stellvertretungsrecht. In diesen Zusammenhang gehört auch das elektronische Skript zum Staatshaftungsrecht von Berkemann (vgl jur-pc 1995, S. 3365, enthalten aufider jur-pc CD-ROM zum CD-ROM-Digest). Über die Studienrelevanz derartiger Hilfsmittel wird man sich (heutzutage) schnell einigen können.

Ist z. B. die Frage des sittenwidrigen Ratenkredits ein ausbildungsrelevantes Thema? Wer mit “ja” antwortet (danke! -)))), wird einen jur-pc Schwerpunkt wie den in Heft 7+8/1990 zu schätzen wissen, der Rechtsprechung und Rechenmethoden zu diesem Thema vorstellte (vgl. S. 655-670). Womit im übrigen auch die regelmäßigen Software-Beilagen auf Diskette berührt sind. Als jur-pc ein vergleichbares Rechenthema 1994 noch einmal aufgriff (vgl. Rüßßmann, Restschuldminderung nach $\$ 12$ Abs. 2 des Verbraucherkreditgesetzes, S. 2828-2837) gab es auf der Diskettenbeilage ein voll funktionsfähiges Excel samt einschlägiger Rechenblätter.

Nicht unberücksichtigt blieben auch Hardware-Komponenten, die man beim "Ausbildungscomputing" nützlich verwenden kann(vgl. z. B. Michel zu einem CD-ROM-Laufwerk für die parallele Schnittstelle, jur-pc 1992, 1764-1765; oder Kraft zu einem LCD-Tablett für's Pen-Computing, jur-pc 1993, S. 2331-2333; oder Höck zu CD-ROM Laufwerken allyemein, jur-pc 1994, S. 2580-2581; oder Sommer zu einem "Lesestift", jur-pc 1994, S. 2946-2951). Allerdings ist in dieser Abteilung im Rückblick schmerzlich die Wandelbarkeit der'Dinge gerade aufidiesem Sektor zu spïren.

In letzter Zeit ist auch das Internet zum Lern- und Ausbildungsinstrument geworden. Dementsprechend widmet jur-pc in Verbindung mit dem Saarbrücker juristischen InternetProjekt (Adresse: http://wwwejura.uni-sb.de) dieser Entwicklung besondere und dauerhafte Aufmerksamkeit. Der Bogen spannt sich insoweit von Voermaneks Präsentation des "Juristen-Gophers" (schon in jur-pc 1993, S. 2286-2292, als das Internet noch nicht in aller Munde war) bis hin zum Beitrag Dippelhofers in diesem Heft.

Die referierten Beispiele stehen für ein Programm. Sicher läßt sich noch einiges tun, um die elektronischen Aspekte der Juristenausbildung flächendeckend zu begleiten, und das gehört fest zum jur-pc-Konzept. Trotzdem war die eingangs referierte Antwort wohl jetzt schon vertretbar. Jedenfalls wird derjenige, der die zitierten Beiträge liest, einen nützlichen Kompaktkurs in Sachen Rechtsinformatik absolvieren. Wenn man im Semester nicht dazu kommt, kann man sich das für die Semesterferien vornehmen.

iurista calculat

Nützliche Hardware

Internet

Gersweiler, den 17. März 1996

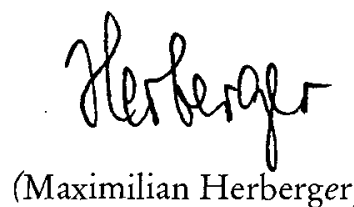

